\title{
INTRODUCTION \\ International women leaders in neurosurgery: past, present, and future
}

\author{
Gail L. Rosseau, MD, ${ }^{1}$ Shelly D. Timmons, MD, PhD, ${ }^{2}$ Karin M. Muraszko, MD, ${ }^{3}$ \\ Katharine J. Drummond, MD, ${ }^{4}$ Mary Murphy, MD, ${ }^{5}$ Nelci Zanon, MD, PhD, ${ }^{6}$ and Farideh Nejat, MD ${ }^{7}$ \\ 'Department of Neurosurgery, George Washington University School of Medicine and Health Sciences, Washington, DC; \\ 2Department of Neurological Surgery, Indiana University School of Medicine and Indiana University Health, Indianapolis, Indiana; \\ ${ }^{3}$ Department of Neurological Surgery, University of Michigan, Ann Arbor, Michigan; " $D$ Department of Neurosurgery, University of \\ Melbourne, Victoria, Australia; ${ }^{5}$ National Hospital for Neurology and Neurosurgery, University College London Hospitals, NHS \\ Foundation Trust, London, United Kingdom; ${ }^{6}$ Pediatric Neurosurgery, Pediatric Neurosurgical Center, São Paulo, Brazil; and \\ ${ }^{7}$ Department of Neurosurgery, Tehran University of Medical Science, Tehran, Iran
}

$\mathrm{I}$ $\mathrm{N}$ this issue of Neurosurgical Focus, women's roles in neurosurgery over the years are explored. While such a broad topic is beyond the scope of a single issue, the papers herein represent a variety of reports on both the impact of culture and history on women's progress and the global influence of women on the field. Topics include academic productivity and the influence of both barriers and mentorship. Various aspects of research are described, including funding gaps and publication rates. The presence of women neurosurgeons at the podium at scientific and society gatherings is also explored, along with the emerging roles of women as leaders in international organizations. The challenges of increasing the visibility of worthy participants in all professional endeavors are discussed. Further, various facets of a changing professional workplace are reported, including parental leave and other wellness policies of interest to both men and women entering neurosurgery, perceptions about the demands of the discipline, and the impact of role models, harassment, and other issues. Historical reports highlight several noteworthy examples of women neurosurgeons around the globe who have demonstrated vision and leadership. By emphasizing the contributions of these distinctive leaders, this issue illuminates the innovation and excellence that can uniquely be achieved by promoting inclusiveness.
Perhaps most importantly, strategies to expand opportunities for success for women neurosurgeons are proposed. Our patients benefit from having neurosurgeons who mirror the populations we serve. A future that actively encourages the best and brightest medical students to choose neurosurgical careers-regardless of gender, ethnicity, religion, culture, sexual orientation or identity, socioeconomic strata, or any other individual defining characteristic-will best serve the needs of our profession and our patients. This issue will allow readers to take stock of progress to date and to redouble efforts for sustainable programs that promote equity and diversity in our specialty.

https://thejns.org/doi/abs/10.3171/2020.12.FOCUS201088

\section{Disclosures}

The authors report no conflict of interest.

\section{Correspondence}

Shelly D. Timmons: stimmons@mac.com. 